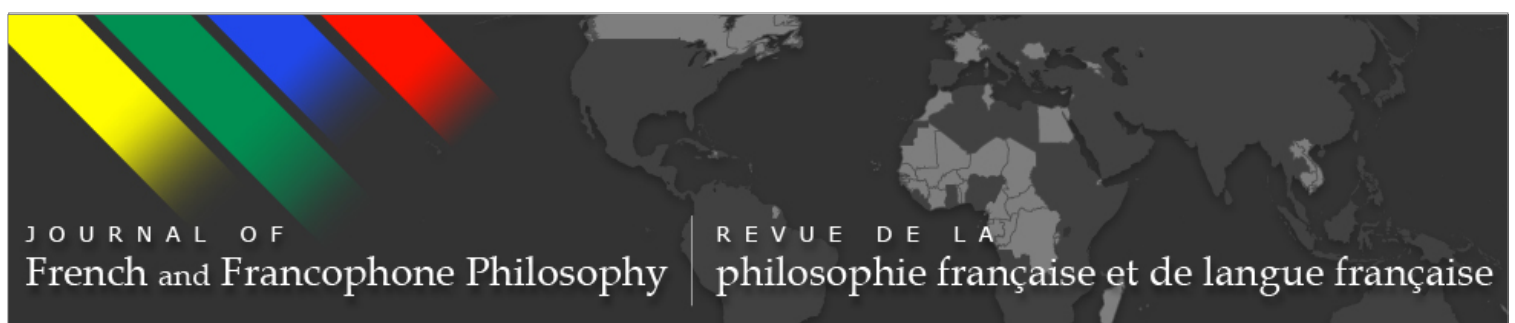

\title{
Créoliser Marx avec Ngugi Wa Thiong'o
}

\section{Sonia Dayan-Herzbrun}

Journal of French and Francophone Philosophy - Revue de la philosophie française et de langue française, Vol XXV, No 2 (2017) pp 45-53.

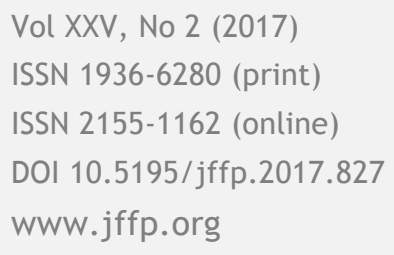

\section{(cc) BY-No-ND}

This work is licensed under a Creative Commons Attribution-Noncommercial-No Derivative Works 3.0 United States License.

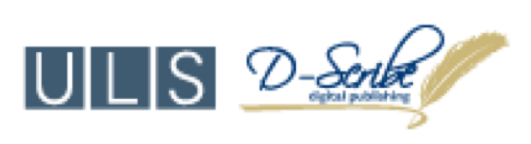

This journal is operated by the University Library System of the University of Pittsburgh as part of its D-Scribe Digital Publishing Program, and is co-sponsored by the University of Pittsburgh Press 


\title{
Créoliser Marx avec Ngugi Wa Thiong'o
}

\author{
Sonia Dayan-Herzbrun
}

Université Paris Diderot

En mettant en question l'utilisation par les colonisés de la langue des colonisateurs et en appelant au retour aux langues africaines, l'écrivain kényan Ngũgĩ wa Thiong'o a produit sans doute la critique la plus radicale et la plus audacieuse qui soit, de la colonisation de l'esprit. ${ }^{1}$ Il ne s'agit pas seulement pour lui de transformer le rapport au savoir. Il veut également libérer l'imaginaire et la sensibilité des chaînes de la colonisation et de l'impérialisme et rendre possible un nouvel agir.

La décision que prend au début des années 1970 celui qui est alors professeur de littérature à l'université de Nairobi, d'écrire désormais en Gikuyu, pourrait être interprétée comme une forme de nativisme. Il la présente, cependant, non comme un retour mais comme une véritable “coupure épistémologique." ${ }^{2}$ On reconnaît ici la notion utilisée par Louis Althusser pour marquer la rupture entre un jeune Marx idéologue, et un Marx fondateur d'une véritable discipline scientifique nouvelle: le marxisme. ${ }^{3}$ Cependant, à l'inverse de ce que propose Althusser, la coupure épistémologique qu'introduit Ngũgĩ consiste à se défaire de l'autorité de la science dite moderne, et donc à se déconnecter de l'univers mental aliéné dans lequel la colonisation a plongé les colonisés et qui est celui de la rationalité instrumentale. ${ }^{4}$ Sa démarche, d'abord personnelle, lui permet de refaire lien avec sa subjectivité, son histoire, sans revenir à une prétendue pureté originelle. En sortant d'une scientificité normale et normée, Ngũgĩ libère donc le concept althussérien de sa pesanteur scientiste et positiviste pour lui donner une portée pleinement émancipatrice. Loin d'être un contresens, cet usage dissonant est une des facettes de la créolisation de Marx, et des multiples figures de ce que l'on a appelé le marxisme, qui s'opère à travers l'œuvre multiforme de Ngũgĩ.

On a, dans une intention bien évidemment malveillante, qualifié Ngũgĩ de marxiste. ${ }^{5}$ Cette qualification se veut disqualification. Elle s'accompagne d'accusations de dogmatisme adressées à un opposant

Journal of French and Francophone Philosophy | Revue de la philosophie française et de langue française Vol XXV, No 2 (2017) | www.jffp.org | DOI 10.5195/jffp.2017.827 
politique notoire par des universitaires qui ont de toute évidence passé avec le pouvoir dictatorial d'Arap Moi les compromis nécessaires à l'obtention de leurs postes. ${ }^{6}$ Or la lecture que fait Ngũgĩ de Marx, de certains auteurs dits marxistes, mais aussi de beaucoup d'autres, loin d'être dogmatique et univoque, est largement hétérogène et hétérodoxe. Elle correspond très précisément à certains des caractères de la créolisation dégagés par Jane Anna Gordon et élaborés dans la Caribbean Philosophical Association. ${ }^{7}$ Comme Gordon l'a fait pour Rousseau, ou Michael Monahan à propos de Hegel, créoliser un penseur venu du Nord global, consiste à en proposer une lecture permettant de mettre en lumière des dimensions qui le désoccidentalisent. $^{8}$ La créolisation fait alors apparaître ce qui, chez ces penseurs, ouvre à un universel en construction, en évolution, dans un mouvement de va et vient où l'Europe perd sa centralité, et où les rapports hégémoniques imposés par la colonisation tendent à s'effacer. La créolisation rend sa liberté à la pensée.

Comme le personnage éponyme du très beau roman de Ngũgĩ, Wizard of the Crow, tantôt homme tantôt oiseau, le Marx de Ngũgĩ s'échappe dès lors qu'on veut le mettre en cage. ${ }^{9}$ La pensée de Marx prend alors un relief nouveau, une portée qui va très au-delà de la lettre du texte de Marx, dont on a beaucoup dit, et à juste titre, qu'il n'avait pas pris la mesure de la colonisation, considérant, comme dans son célèbre article The British Rule in India, paru en juin 1853 dans le New York Herald Tribune, la conquête coloniale, avec toutes les horreurs qui l'accompagnent, comme un mal nécessaire pour entrer véritablement dans l'histoire. "Whatever may have been the crimes of England she was the unconscious tool of history in bringing about that revolution," écrit-il dans la conclusion de cet article. Pour que la pensée de Marx et de ceux qui se sont référés à lui, retrouve une force émancipatrice capable de résister à ce que le philosophe Joseph Tonda analyse comme un "impérialisme postcolonial" et à la violence de l'imaginaire dont il use et abuse, il fallait bien qu'elle soit créolisée.

Tout commence comme dans un conte. Un matin de 1976 une femme arrive chez Ngũgĩ. Elle vient d'un village voisin, Kamiriithu, chez celui qui est alors déjà un écrivain connu, un afro-saxon comme il le dira, et directeur du département de littérature de l'université de Nairobi. Elle lui demande de consacrer un peu de son temps à l'éducation et à la culture des villageois, et pas seulement à celle des étudiants issus de la bourgeoisie. Il commence par refuser. Elle reviendra quatres dimanches de suite jusqu'à ce qu'il accepte. Le cours de son existence en sera changé, à la fois interrompu et réunifié. C'est avec les villageois eux-mêmes qu'il choisira la forme que prendra cette entreprise: le théâtre. Un théâtre qui reprendra et donnera une vie nouvelle à ce qui était propre au théâtre africain: le chant et la danse. Les villageois seront co-auteurs, acteurs. Les pièces écrites par Ngũgĩ leur permettront de raconter et d'analyser leur propre histoire, dans leur langue vernaculaire, le Gikuyu.

Journal of French and Francophone Philosophy | Revue de la philosophie française et de langue française Vol XXV, No 2 (2017) | http://www.jffp.org | DOI 10.5195/jffp.2017.827 
Ils ont, en effet, beaucoup à dire, et d'abord sur tout ce dont ils ont été dépossédés. Il faut leur rendre la parole, leur parole, et inventer des formes d'expression d'où ils ne soient pas exclus; à côté de la littérature, rendre toute sa dignité à l'"'orature," car l'écriture est imposition de pouvoir et mise à distance de la subjectivité. ${ }^{10}$ En effet c'est aussi de leur rapport à euxmêmes que ces villageois ont été dépouillés. Ngũgĩ n'a cessé de rappeler que ce ne sont pas les ouvriers, les paysans, les femmes des classes populaires qui ont de fait mené la lutte contre la colonisation, et souvent au prix de souffrances extrêmes, ${ }^{11}$ qui en ont tiré les bénéfices, mais un groupe de privilégiés qui ne sont, à bien les regarder, que des voleurs et des malfrats: "Power has been taken away from the workers and peasants and given to the heroes of theft and robbery-in English, we might say, to those who have capitalist business know-how." 12

Ici une remarque s'impose. Le roman Devil on the Cross d'où est extraite cette citation est écrit en Gikuyu et traduit en anglais par Ngũgĩ luimême, qui met en italiques les expressions rédigées en anglais. L'anglais n'est donc plus la langue de référence, mais une langue étrangère à laquelle on peut avoir recours en cas de besoin et de façon très limitée, notamment pour exprimer de façon abstraite ce qui est éprouvé dans l'expérience et décrit comme tel en Gikuyu, avec toute sa charge émotionnelle. Il y a là comme une enchevêtrement, une imbrication qui est le propre de la créolisation, comme le signale Jane Gordon: "La créolisation montre une manière fondamentalement poreuse et imbriquée de comprendre les sujets humains, les relations et les mondes." ${ }^{13}$ Quand des habitants de Kamiriithu qui ont participé à la guérilla des Mau Mau montent une représentation théâtrale, ils veulent que leur vie de travailleurs à l'usine de chaussures Bata y soit racontée. Et quand ils expliquent qu'ils n'étaient rémunérés que pour une journée de travail alors qu'ils travaillaient douze heures par jour, un mois durant, c'est très précisément du processus d'extraction de la plusvalue, qu'ils parlent, c'est à dire de "cette partie de la valeur totale d'une marchandise dans laquelle se trouve réalisé le surtravail ou travail non payé $\mathrm{d}^{\prime}$ un ouvrier" ${ }^{14}$ et qui apparaît comme profit. On est bien là dans ce que Jane Gordon conceptualise comme "cross-breeding,"15 c'est à dire ce mouvement réciproque par lequel des pensées politiques-ici la réflexion des anciens résistants Mau Mau et la théorie marxiste-s'alimentent l'une l'autre, ouvrant des possibilités inédites de praxis émancipatrice.

Les mots de Ngũgĩ sont comme un pont entre des expériences et des modes d'expression divers. Marx a montré comment cet apparent "échange entre le capital et le travail" qui a "pour résultat constant de reproduire le travailleur comme travailleur, et le capitaliste comme capitaliste," ${ }^{16}$ peut transformer les travailleurs, s'ils se soumettent et se résignent, à n'être plus qu'une masse affamée d'"écrasés désormais incapables de salut." ${ }^{17}$ C'est pourquoi il les appelle à lutter non pas pour l'amélioration toujours illusoire de leur salaire, mais pour l'abolition du salariat. Ngũgĩ parle lui de "lutte

Journal of French and Francophone Philosophy | Revue de la philosophie française et de langue française Vol XXV, No 2 (2017) | http://www.jffp.org | DOI 10.5195/jffp.2017.827 
entre capital et travail" ${ }^{18}$ comme étant le thème central du long monologue d'une des personnages de Ngaahika Ndeenda, (I will marry when I want), la pièce qu'écrit et que monte Ngũgĩ à Kamiriithu. Mais d'emblée les termes empruntés au vocabulaire marxiste le plus classique, prennent dans le contexte kényan, une toute autre portée.

La lutte dont il est ici question est menée par les ouvriers et les paysans, dont Marx considérait, à la lumière de l'expérience européenne, et surtout française qu'ils avaient été réduits à n'être qu'un groupe conservateur. ${ }^{19}$ Créoliser Marx, c'est ici donner toute leur place et toute leur dignité à ces acteurs. Le combat mené a été un combat pour la terre et pour la liberté, quand il s'agissait de conquérir l'indépendance du Kenya, non pas pour transformer la terre en parcelles de propriété privée, mais pour en réinventer, ensemble, un usage collectif. Là encore, il y a comme un aller et retour entre l'horizon utopique des différentes composantes du marxisme et les valeurs et façons de vivre du monde Gikuyu d'avant la colonisation: "As Gikuyu once said, a single finger cannot kill a louse; a single log cannot make a fire last through the night; a single man, however strong, cannot build a bridge across a river; and many hands can lift a weight, however heavy." 20

Les femmes-elles aussi des actrices à peu près totalement absentes de l'orthodoxie marxiste européenne-jouent un rôle extrêmement actif dans le récit de cette lutte que fait Ngũgĩ, parce que les rapports capitalistes pèsent sur elles lourdement et de façon spécifique. Mais elles sauront se battre. Jeunes et belles les femmes subissent violences et harcèlement sexuels. Agées et pauvres, elles sont mises au rebut. Wariinga, l'héroïne de The Devil on the Cross, perd son travail de secrétaire quand elle refuse les avances de son patron, donc ses revenus et son logement, et son amoureux la rejette parce qu'il la croit infidèle. De retour dans son village, elle finira par abattre le "vieil homme riche" qui jadis l'avait séduite, alors qu'elle n'était qu'une adolescente, puis l'avait abandonnée quand elle était tombée enceinte. Au début du roman elle était accablée, passive, elle ne songe qu'à se suicider. La rencontre de militants lui permettra de décrypter le sens politique de sa situation, de ne plus la vivre comme une fatalité, et de devenir une combattante. En guise d'oraison funèbre elle dit avec mépris de celui qu'elle vient de tuer: "There kneels a jigger, a louse, a weevil, a flea, a bedbug! He is a misteltoe, a parasite that lives on the trees of other people lives." ${ }^{21} \mathrm{Et}$ quand elle part, personne ne peut l'arrêter. D'une certaine manière, elle aussi a pris son vol. L'exercice de la liberté est agonistique, chez Ngũgĩ, comme chez Marx ou chez Fanon.

C'est qu'il ne s'agit pas seulement de mettre fin au capitalisme, mais aussi à l'impérialisme, qui en est le "stade suprême," comme l'écrivait Lénine dans le livre L'impérialisme, stade suprême du capitalisme, dont Ngũgĩ recommandait la lecture à ses étudiants, quelques jours avant d'être arrêté et incarcéré en raison de ses activités à Kamiriithu. L'indépendance nationale

Journal of French and Francophone Philosophy | Revue de la philosophie française et de langue française Vol XXV, No 2 (2017) | http://www.jffp.org | DOI 10.5195/jffp.2017.827 
proclamée en 1963 n'avait pas mis fin à l'impérialisme. Elle lui avait donné un nouveau visage. C'est pourquoi, en même temps que Lénine, Ngũgĩ conseillait à ses étudiants de lire le chapitre III des Damnés de la Terre de Frantz Fanon: "Mésaventures de la conscience nationale." À lire Ngũgĩ, on ne peut que penser à cette phrase quasi prophétique de Fanon:

Avant l'indépendance, le leader incarnait en général les aspirations du peuple: indépendance, libertés politiques, dignité nationale; mais au lendemain de l'indépendance, loin d'incarner concrètement les besoins du peuple, loin de se faire le promoteur de la dignité réelle du peuple, celle qui passe par le pain, la terre et la remise du pays entre les mains sacrées du peuple, le leader va révéler sa fonction intime: être le président-général de la société des profiteurs impatients de jouir que constitue la bourgeoisie nationale. $^{22}$

L'indépendance, pour laquelle des milliers de Kényans étaient morts, a été l'objet d'un détournement. À la colonisation a succédé un impérialisme beaucoup plus vaste et complexe. Le Kenya est devenu "a neo-colony with the doors open to wider imperialist interests from Japan to America." ${ }^{23}$ Dans ce capitalisme mondialisé, nom savant du braquage et du vol, les malfaiteurs opèrent chez eux et en dehors de chez eux. Au Kenya, ils sont aidés par des malfrats locaux qui récoltent les bénéfices qu'ils peuvent, en richesses, en prébendes, bien sûr, mais aussi en exercice effréné du pouvoir et en prestations sexuelles obtenues de gré ou de force. "Today, for instance, American, European and Japanese thieves and robbers steal from their own masses, and then go on to Africa, Asia and Latin America to rob the peoples there, and take their plunder back to their own granaries. These foreigners are, of course, aided in their enterprise by bands of local thieves and robbers." 24

Les mots et les images qu'utilise Ngũgĩ sont accessibles à tous. Le Marx qui fréquentait chaque jour la British Library était empêtré dans un langage qui se voulait scientifique. Il le reconnaissait lui-même. Quand il demande à Ferdinand Lassalle qui vit alors à Berlin de trouver un éditeur pour son nouveau livre qui porte sur "le système de l'économie bourgeoise exposé de manière critique" ${ }^{25}$ et qui est connu sous le titre de Critique de l'économie politique, il précisera: “L'exposé, je veux dire la manière, est tout à fait scientifique, c'est-à-dire absolument pas contraire aux règlements de police au sens habituel." 26 Ce choix d'une "manière scientifique" lui permet, certes, d'échapper aux rigueurs de la censure. Mais on voit aussi à quel public familier des sciences économiques il souhaite s'adresser. On soulève parfois la question du lieu social à partir duquel écrivait et théorisait Marx. La question me semble être plutôt celle du public auquel il s'adressait et surtout celle du langage choisi. Un langage d'autorité, parfaitement

Journal of French and Francophone Philosophy | Revue de la philosophie française et de langue française Vol XXV, No 2 (2017) | http://www.jffp.org | DOI 10.5195/jffp.2017.827 
convaincant dès lors qu'on a réussi à y accéder. Mais un langage qui reste celui d'une élite mâle et blanche.

Ngũgĩ créolise Marx en le soumettant en quelque sorte à une transmutation. En lui faisant parler un autre langage et une autre langue. Marx avait montré comment la domination de classe s'exprime et se masque à travers ce qu'il désignait comme idéologie. On ne pouvait combattre l'idéologie qu'en la démasquant à l'aide de la critique, une critique savante, et faire apparaître les rapports d'exploitation et de domination qui la soustendent. Avec sa poésie et surtout son théâtre, Bertolt Brecht à qui Ngũgĩ se réfère souvent, a su donner à entendre et à voir à un public beaucoup plus large et cette fois populaire, la face à la fois cachée et tellement évidente de la violence sociale et économique. Mais de la violence coloniale il n'était pas question. Or la domination et violence coloniales sont également culturelles et épistémiques. Elles le sont, en quelque sorte, doublement: parce qu'elles émanent du capitalisme et du fait d'une spécificité coloniale.

Colonialism imposed its control of the social production of wealth through military conquest and subsequent political dictatorship. But its most important area of domination was the mental universe of the colonised, the control through culture of how people perceived themselves and their relationship to the world. Economics and political control can never be complete or effective without mental control. To control a people's culture is to control their tools of self-definition in relationship to others. ${ }^{27}$

En d'autres termes, le colonialisme interdit aux colonisés l'expression de leur propre subjectivité, de leur propre manière d'être dans le monde. C'est l'école et l'inculcation de la langue des colonisateurs qui vont être les véhicules de cette interdiction. Après la conférence de Berlin de 1884-1885, L'Afrique, après avoir été divisée en Afrique du Nord et Afrique subsaharienne, va être répartie en grandes zones soumises à l'impérialisme culturel de telle ou telle puissance et donc de telle ou telle langue: anglophone, lusophone, francophone. L'expérience propre des colonisés, leur histoire, leur univers symbolique, vont être au mieux dévalorisés ou stigmatisés, au pire effacés. L'unité et la cohésion interne des sociétés colonisées vont être brisées. Il y aura d'un côté les plus démunis sur lesquels pèseront lourdement domination et exploitation, sans parler des violences politiques extrêmes. De l'autre il y aura une bourgeoisie néocoloniale qui singe les colonisateurs. "Their ideas are spread by a corpus of state intellectuals, the academic and journalistic laureates of the neo-colonial establishment." $^{28}$ Ces intellectuels soumis aux États autoritaires post et néocoloniaux ont intériorisé la conviction de la supériorité de la langue du colonisateur. Ce sont des figures de ce que le jeune Marx nomme "aliénation." En France on les récompense en leur décernant des prix littéraires prestigieux ou en les faisant entrer à l'Académie Française. Ngũgĩ

Journal of French and Francophone Philosophy | Revue de la philosophie française et de langue française

Vol XXV, No 2 (2017) | http://www.jffp.org | DOI 10.5195/jffp.2017.827 
n'est pas tendre avec eux. ${ }^{29}$ D'autres, rebelles, ont travaillé la langue du colonisateur, de façon à la rendre apte à transmettre leur expérience d'Africains ou de Caribéens, irrigant la langue imposée de la force de la langue de leur enfance. C'est sans doute la parfaite maîtrise que Ngũgĩ a de la langue anglaise qui lui permet, en devenant son propre traducteur, de parler et d'écrire depuis le lieu dans lequel il est advenu au monde, et d'échapper au localisme dans lequel ses détracteurs tendraient à l'enfermer.

Lutter contre l'impérialisme sous ses formes successives c'est revenir à son histoire, à son expérience. Le sujet s'éprouve dans un nous, et pour Ngũgĩ, ce nous est celui de la famille de paysans dans laquelle il est né, dans laquelle il a grandi, et qui parlait le Gikuyu. "The home and the field were then our pre-primary school but what is important, is that the language of our evening teach-ins, and the language of our immediate and wider community, and the language of our work in the fields were one." On comprend dès lors mieux pourquoi Ngũgĩ décide de monter à Kamiriithu des spectacles politiques en Gikuyu en revenant aux formes narratives et aux expressions corporelles du théâtre africain dans le village de Kamiriithu. Mais le privilège qu'il accorde personnellement au Gikuyu, parmi les autres langues parlées au Kénya, outre qu'il lui permet de refaire lien avec son expérience, a aussi un sens politique. La région Gikuyu est celle où la résistance à la colonisation britannique a été la plus forte. Dans les centres de détention britanniques, les missionnaires, dans leurs prêches, demandaient aux prisonniers, des Gikuyus, de rejeter les Mau Mau en les diabolisant. “Did the British think we didn't see this hyprocrisy? These men preaching Christian brotherhood were some of the same ones fighting against us and benefiting from our losses," dit un ancien détenu. ${ }^{30}$ À l'inverse de l'effet escompté, on a assisté à un regain dans la résistance, des croyances propres aux Gikuyu. Après l'indépendance, comme le rappelle à des très nombreuses Ngũgĩ, les Gikuyus sont devenus suspects, du fait même de leur force de résistance. Le swahili, une langue commune à plusieurs régions d'Afrique de l'Est, est privilégié, même si au niveau institutionnel et académique, c'est l'anglais qui s'impose. Et c'est en raison de ses activités d'homme de théâtre profondément engagé dans le village de Kamiriithu, que Ngũgĩ sera arrêté en décembre 1977 et détenu un an de façon arbitraire, sans jamais passer devant un tribunal.

Ngũgĩ a donné vie au Centre culturel communautaire de Kamiriithu, en conversation avec ces très grands dramaturges qu'ont été Peter Brook et Augusto Boal. La référence à Peter Brook et à sa conception d'un "espace vide" $^{31}$ est particulièrement intéressante. En effet Peter Brook a fait de très nombreux et très longs séjours en Afrique et la manière dont il a peu à peu conçu son rapport au public et à l'espace théâtral (sans décors, comme il l'avait vu faire dans les villages africains) doit très largement à l'Afrique. Le retour au Gikuyu n'implique nullement un repli ou un enfermement; c'est la condition de possibilité d'une universalisation à partir de la reconnaissance

Journal of French and Francophone Philosophy | Revue de la philosophie française et de langue française Vol XXV, No 2 (2017) | http://www.jffp.org | DOI 10.5195/jffp.2017.827 
de pluralités en mouvement. Ngũgĩ cite abondamment la littérature occidentale ainsi que la littérature africaine, celle du continent et celle des diasporas. Il les connaît toutes parfaitement et les fait dialoguer.

En assumant ce qui lui est propre en tant qu'Africain, dans une région particulière de l'Afrique, il recouvre son humanité et celle des autres, c'est à dire toute l'humanité. De la lutte culturelle qu'il mène contre l'impérialisme néocolonial, il écrit "It is part of that struggle for that world in which my health is not dependent on another's leprosy; my cleanliness not on another's maggot-ridden body; and my humanity not on the buried humanity of others." ${ }^{32}$ Et c'est à ce niveau là qu'il retrouve Marx, non pas le Marx scientifisé par Althusser, mais celui dont ne voulait pas Althusser, le jeune Marx qui, lors d'un séjour à Paris en 1844, jetait sur des cahiers les réflexions qui lui venaient sur les lectures du moment. C'est un Marx qui parlait d'humanité, d'art et d'amour. "Imagine l'homme humain et son rapport au monde comme un rapport humain, et tu ne pourras échanger l'amour que contre l'amour, la confiance contre la confiance, etc." ${ }^{33} \mathrm{C}^{\prime}$ est ce même Marx qui appelait, l'année suivante, dans sa célèbre onzième thèse sur Feurbach, les philosophes qui jusqu'alors s'étaient contenté de penser le monde, à le transformer afin que justement le rapport au monde redevienne un rapport humain. Ngũgĩ se fait l'écho de cet appel et le relaie en direction de tous les "damnés de la terre," qu'ils se trouvent en Afrique, en Asie ou en Amérique Latine. Marx, le "Maure" comme on le surnommait, est ainsi définitivement créolisé, et sa pensée, à être relue, nourrie d'expériences et d'histoires inédites et inattendues, peut inciter à inventer des pratiques de libération bien au-delà de tout ce que lui-même aurait pu imaginer.

${ }^{1}$ Ngũgĩ wa Thiong'o, Decolonising the Mind: The Politics of Language in African

Literature (Portsmouth, NH: James Currey/Heinemann, 1986).

2 Thiong'o, Decolonising the Mind, 44.

${ }^{3}$ Louis Althusser, Pour Marx (Paris: François Maspéro, 1967), 24.

${ }^{4}$ Thiong'o, Decolonising the Mind, 57.

${ }^{5}$ B.A. Ogot and W.R. Ochieng, Decolonization \& Independence in Kenya (Athens, OH: Ohio University Press, 1995), 232.

${ }^{6}$ Kowar G. Adar and Isaac M. Munyae, "Human Rights Abuse in Kenya under Daniel Arap Moi, 1978-2001," African Studies Quarterly 5, no. 1 (Winter 2001): 1-17.

7 Jane Anna Gordon, "Creolizing as the Transdisciplinary Alternative to Intellectual Legitimacy on the Model of the 'Normal Scientific' Community," Quaderna, mis en ligne le 21 janvier 2016: http://quaderna.org/.

Journal of French and Francophone Philosophy | Revue de la philosophie française et de langue française Vol XXV, No 2 (2017) | http://www.jffp.org | DOI 10.5195/jffp.2017.827 
8 Jane Anna Gordon, Creolizing Political Theory: Reading Rousseau through Fanon (New York: Fordham University Press, 2014) et Michael Monahan, ed., Creolizing Hegel (London: Rowman and Littlefield International, 2017).

${ }^{9}$ Ngũgĩ wa Thiong'o, Wizard of the Crow (New York: Anchor Books, 2006).

${ }^{10}$ Thiong'o, Decolonising the Mind, 77-78.

${ }^{11}$ Caroline Elkins, Imperial Reckoning: The Untold Story of Britain's Gulag in Kenya (New York: Henry Holt and Company, 2006).

${ }^{12}$ Ngũgĩ wa Thiong'o, Devil on the Cross (Portsmouth, NH: Heineman, 1982), 165.

13 Jane Anna Gordon, "Par-delà la seule critique. Créoliser nos entreprises intellectuelles et politiques," Revue Tumultes, $\mathrm{n}^{\circ} 48$ (2017): 30.

${ }^{14}$ Karl Marx, Salaire, prix et plus-value, dans Karl Marx, CEuvres, tome 1 (Paris : Bibliothèque de la Pléiade, 1965 [1865]), 516.

${ }^{15}$ Gordon, Creolizing Political Theory, 197.

${ }^{16}$ Marx, Salaire, prix et plus-value, 513.

${ }^{17}$ Marx, Salaire, prix et plus-value, 532.

18 Thiong'o, Decolonising the Mind, 55.

${ }^{19}$ Karl Marx, Le dix-huit brumaire de Louis Bonaparte (1852).

20 Thiong'o, Devil on the Cross, 52.

21 Thiong'o, Devil on the Cross, 254.

${ }^{22}$ Frantz Fanon, Les damnés de la terre (Paris: La Découverte, 2002 [1961]), 160.

${ }^{23}$ Thiong'o, Decolonising the Mind, 44.

24 Thiong'o, Devil on the Cross, 165.

25 “Lettre de Marx à Lassalle du 22 février 1858," in Ferdinand Lassalle and Karl Marx, Correspondance (1848-1854), présentation et traduction par S.Dayan-Herzbrun (Paris: Presses Universitaires de France, 1977), 154.

${ }^{26}$ Marx, “Lettre de Marx à Lassalle du 22 février 1858," 154.

27 Thiong'o, Decolonising the Mind, 16.

28 Thiong'o, Decolonising the Mind, 16.

${ }^{29}$ Thiong'o, Decolonising the Mind, 18-19.

${ }^{30}$ Elkins, Imperial Reckoning, 173.

31 Peter Brook, The Empty Space: A Book About the Theatre: Deadly, Holy, Rough, Immediate (New York: Torchstone, 1968).

${ }^{32}$ Thiong'o, Decolonising the Mind, 106.

33 Karl Marx, "Économie et philosophie" (Manuscrits parisiens), dans OEuvres, tome 2 (Paris, Bibliothèque de la Pléiade, 1968 [1844]), 118. Cité par Thiong’o, Decolonising the Mind, 107.

Journal of French and Francophone Philosophy | Revue de la philosophie française et de langue française Vol XXV, No 2 (2017) | http://www.jffp.org | DOI 10.5195/jffp.2017.827 